\title{
Trypanocidal Activity and Increased Solubility of Benznidazole Incorporated in PEG 4000 and Its Derivatives
}

\author{
Lucas R. D. Sousa, ${ }^{a}$ Maria Luiza S. Azevedo, ${ }^{a}$ Dayana F. Rocha, ${ }^{a}$ Angela L. Andrade, ${ }^{a}$ \\ Tatiane R. Amparo, ${ }^{b}$ Orlando D. H. dos Santos, ${ }^{b}$ Janaína B. Seibert, ${ }^{c}$ Luciano R. Pereira, ${ }^{c}$ \\ Paula M. A. Vieira, ${ }^{d}$ Cláudia M. Carneiro, ${ }^{b}$ Ana Paula M. Barboza, ${ }^{e}$ Bernardo R. A. Neves, ${ }^{f}$ \\ Policarpo A. Sales Jr., ${ }^{g}$ Silvane M. F. Murta, ${ }^{g}$ Kátia M. Novack ${ }^{a}$ and \\ Viviane M. R. dos Santos ${ }^{\circledR} *, a$ \\ ${ }^{a}$ Departamento de Química, Instituto de Ciências Exatas e Biológicas, \\ Universidade Federal de Ouro Preto, 35400-000 Ouro Preto-MG, Brazil \\ ${ }^{b}$ Escola de Farmácia, Universidade Federal de Ouro Preto, 5400-000 Ouro Preto-MG, Brazil

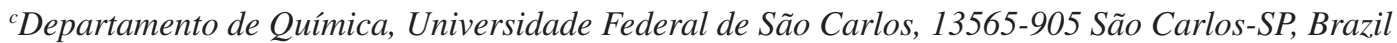 \\ ${ }^{d}$ Departamento de Ciências Biológicas, Universidade Federal de Ouro Preto, \\ 35400-000 Ouro Preto-MG, Brazil \\ ${ }^{e}$ Departamento de Física, Instituto de Ciências Exatas e Biológicas, \\ Universidade Federal de Ouro Preto, 35400-000 Ouro Preto-MG, Brazil \\ ${ }^{f}$ Departamento de Física, Universidade Federal de Minas Gerais, 31270-901 Belo Horizonte-MG, Brazil \\ ${ }^{8}$ Instituto René Rachou, Fundação Oswaldo Cruz (Fiocruz), 30190-002 Belo Horizonte-MG, Brazil
}

\begin{abstract}
Selecting a polymer depends on its characteristics, the properties of the drug and of the remaining ingredients in the formulation. The drug, when incorporated into a polymeric matrix, can show several advantages when compared with its conventional form. In this context, this work describes the preparation and characterization of polyethylene glycol (PEG 4000) and its derivative particles loaded with benznidazole, as well as evaluates their trypanocidal activity. In this work, reactions to modify the PEG 4000 polymer and the subsequent incorporation of the benznidazole were made. The nuclear magnetic resonance (NMR) analysis confirmed the efficiency in modifying the PEG chains. The morphology of polymeric films was observed by atomic force microscopy (AFM) and showed considerable changes on the film organization. The acetylation of PEG favored the stability of the system and an increase in the zeta potential from -14.83 to $-25.54 \mathrm{mV}$ was observed. Although encapsulation efficiency values between 30.14 and $39.48 \%$ were found, the enhanced benznidazole dissolution profile by microparticles enables the use of lower drug concentrations. This fact can be proven by the increased trypanocidal effect of benznidazole when encapsulated in BP3 microparticles. Finally, the high selectivity of the formulations for trypanocidal action guarantees their safety as an alternative for the treatment of the Chagas disease.
\end{abstract}

Keywords: benznidazole, Chagas disease, cytotoxicity, microparticles, polymer

\section{Introduction}

Chagas disease $(\mathrm{CD})$ is an endemic protozoonosis in Latin America, caused by the hemoflagen protozoan Trypanosoma cruzi. ${ }^{1}$ This disease is considered neglected by the World Health Organization., ${ }^{2,3}$ The benznidazole and nifurtimox are the medications available for the treatment of

*e-mail: vivianesantos@ufop.edu.br
CD worldwide, however, they have important adverse effects that lead patients to abandon the treatment. ${ }^{4}$ In general, most drugs used in the chemotherapy of neglected tropical diseases (NTDs) are poorly soluble in water. ${ }^{5}$ Although it is not the ideal drug of choice, due to its low solubility and consequently high toxicity, benznidazole is currently the only drug available for the treatment of CD in Brazil. ${ }^{6}$

Drugs with high toxicity, when incorporated in polymeric matrices, can produce less adverse effects. 
Recently, many polymers have been developed to deliver drugs to target places. There is a great diversity of systems, such as liposomes, osmotic pumps, enteric coating, transdermal systems, pro-drugs, polymeric matrix systems, which provide a drug dissolution with a longer time in the body, especially in the target-tissue or organ, than the conventional, becoming an issue of great interest in the pharmaceutical industries. ${ }^{?}$

Polymers are very versatile for a series of applications including pharmaceutical applications. ${ }^{8}$ Polymeric excipients constitute a very large and varied group of substances, including macromolecular compounds of natural origin, e.g., sodium alginate, gelatin, chitosan and cellulose derivatives; semisynthetic polymers, e.g., cellulose derivatives; synthetic polymers, e.g., polyethylene glycols, poloxamers, polylactides, polyamides and acrylic acid polymers. ${ }^{8}$ Because of their broad range of applications, particularly for the increase of the drug dissolution, polymers are among the most used excipients in pharmaceutical technology. ${ }^{9}$ While classifying specific groups of multifunctional polymers, the advantages of using them when designing a new drug, include increasing substance bioavailability, improving substance stability during formulation and the possibility of obtaining controlled forms of localized dissolution to a specific site in the organism. In order to achieve specific drug dissolution profiles, synthetic polymers may be chemically modified. ${ }^{5}$ Polymers have been used as bioactive pharmaceuticals due to their increased drug efficacy, solubility, and target specificity compared to small-molecule drugs..$^{10}$ The insertion of functional groups in polymer compounds may facilitate their interaction with different drugs. Polyethylene glycol (PEG) polymers are widely used for their low melting point, low toxicity, drug compatibility, and hydrophilicity. ${ }^{11}$

In this context, the increase of poorly water-soluble drugs dissolution can improve its bioavailability, decrease the dose and consequently reduce its adverse effects. Modifications to the PEG 4000 polymer were made by 2 types of chemical modifications for functional groups to be inserted in its polymeric chain to facilitate the incorporation process of benznidazole and, consequently, its dissolution. Microparticles of PEG 4000 and its derivatives containing benznidazole were developed and the increase of its effectiveness against $T$. cruzi from the modified polymers was proven.

\section{Experimental}

All commercial reagents and anhydrous solvents were used as received and purchased from commercial suppliers (Sigma-Aldrich, Corp., St. Louis, USA).

\section{Synthesis of PEG 4000 derivatives}

PEG 4000 derivatives were synthesized by 2 types of chemical modifications (Scheme 1). ${ }^{10-14}$

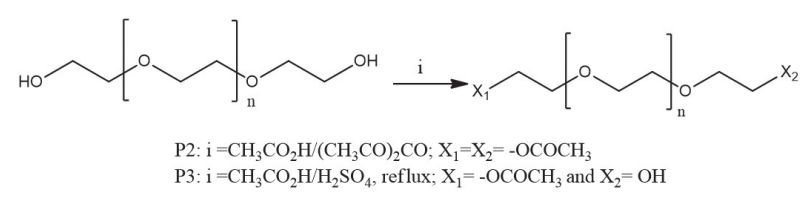

Scheme 1. The general reaction for the synthesis of modified PEG 4000.

Synthesis of acetylated PEG 4000 (P2) ${ }^{14}$

In a round bottom flask, $2.00 \mathrm{~g}$ of PEG 4000 solubilized in $10.00 \mathrm{~mL}$ of dichloromethane and a solution of $5.00 \mathrm{~mL}$ of acetic anhydride and $5.00 \mathrm{~mL}$ of acetic acid were added. The reaction was maintained at a temperature of $40{ }^{\circ} \mathrm{C}$ for $30 \mathrm{~min}$. Upon completion of the reaction, the mixture was poured into a beaker with $100.00 \mathrm{~mL}$ of ice water. The modified PEG 4000 solidified and was separated by simple filtration. Solvent evaporation was made in a stove for $40^{\circ} \mathrm{C}$ and $1.00 \mathrm{~g}$ of a dry white film was obtained.

\section{Synthesis of esterified PEG 4000 (P3) ${ }^{14}$}

In a round bottom flask $2.00 \mathrm{~g}$ of PEG 4000 were solubilized in $10.00 \mathrm{~mL}$ of dichloromethane, $0.10 \mathrm{~mL}$ of concentrated $\mathrm{H}_{2} \mathrm{SO}_{4}$ and $7.00 \mathrm{~mL}$ of $\mathrm{CH}_{3} \mathrm{COOH}$. The reaction was made at a temperature of $40{ }^{\circ} \mathrm{C}$ for $65 \mathrm{~min}$. After cooling, $10 \mathrm{~mL}$ of water were added and the organic phase was dried with anhydrous $\mathrm{MgSO}_{4}$ and filtered. After complete solvent evaporation in a stove for $40{ }^{\circ} \mathrm{C}, 1.50 \mathrm{~g}$ of a dry white film was obtained.

\section{Incorporation of benznidazole in PEG 4000 derivatives}

In order to obtain the polymeric benznidazole particles, $1.20 \mathrm{~g}$ of polymer and $0.40 \mathrm{~g}$ of benznidazole were added to a beaker with $6.00 \mathrm{~mL}$ of acetone at approximately $30^{\circ} \mathrm{C}$. This system was kept mixing until complete dissolution. In a beaker containing $40.0 \mathrm{~mL}$ of water at approximately $70{ }^{\circ} \mathrm{C}, 0.48 \mathrm{~g}$ of polyvinyl alcohol were dispersed, stirring slowly until complete dissolution. The aqueous phase was poured into the organic phase. The mixture was subjected to agitation at $500 \mathrm{rpm}$ for $4 \mathrm{~h}$ at $35^{\circ} \mathrm{C}$. Then, the products were taken to the oven at $40{ }^{\circ} \mathrm{C}$ for $24 \mathrm{~h}$ to evaporate the acetone. After this time, the particles were obtained..$^{10,11}$

\section{Nuclear magnetic resonance (NMR) analysis}

Nuclear magnetic resonance measurements were performed by Bruker spectrometer DRX- 9.4 Tesla (Ettlingen, Germany), equipped with an automatic sampler, 
$5 \mathrm{~mm}$ BFO probe (smart probe with ATMA $\left.{ }^{\oplus}\right)$. The applied field frequency was $100 \mathrm{MHz}$ for carbon. The products were analyzed in dimethyl sulfoxide (DMSO- $d_{6}$ ).

\section{Atomic force microscopy (AFM)}

The samples were characterized using atomic force microscopy (AFM). The AFM data were acquired on a Bruker Multimode 8 SPM (Santa Barbara, USA), using the intermittent contact imaging mode. Silicon cantilevers (NSC35/AlBS from Mikromasch, Tallinn, Estonia) with spring constants of 5-15 $\mathrm{N} \mathrm{m}^{-1}$ and tip radius of curvature ca. $10 \mathrm{~nm}$ were employed throughout this work.

\section{Determination of zeta potential}

The zeta potential of the incorporated benznidazole microparticles was determined by electrophoretic mobility measurements using Zetasizer (Malvern, model Zetasizer Nano series, Nano ZS, Malvern, United Kingdom). The samples were diluted in distilled water and inserted into a capillary cell. The measurements were performed in triplicate for three different samples and the results were expressed in millivolts $(\mathrm{mV}){ }^{15}$

\section{Determination of encapsulation efficiency}

Initially, beznidazole and other constituents that could interfere with its quantification were scanned at intervals of 200 to $400 \mathrm{~nm}$ in spectrophotometer to observe their characteristic absorbance. After preparation, incorporated benznidazole microparticles were dissolved in ethanol and filtered using a cellulose acetate membrane $(0.45 \mu \mathrm{m})$ to separate non-encapsulated drug. Drug content present in the filtrate was quantified in triplicate by measuring the absorbance at $322 \mathrm{~nm} .{ }^{16}$ Encapsulation efficiency (EE) was calculated as follows:

$\mathrm{EE}(\%)=\left(\mathrm{W}_{\mathrm{t}}-\mathrm{W}_{\mathrm{f}} / \mathrm{W}_{\mathrm{t}}\right) \times 100$

where $\mathrm{W}_{\mathrm{f}}$ is the non-encapsulated benznidazole and $\mathrm{W}_{\mathrm{t}}$ is the theoretical benznidazole content in the microparticles.

\section{Determination of dissolution profile}

For the dissolution test, phosphate buffered saline (PBS) solutions at $\mathrm{pH} 3.0$ and 7.3 were prepared. Solutions of each polymer incorporated with benznidazole were prepared and added in these buffer solutions at $37^{\circ} \mathrm{C}$ under agitation. The aliquots of the samples were removed at determined periods (starting from 0 up to a maximum of $72 \mathrm{~h}$ ) and then evaluated in an ultraviolet spectrophotometer at wavelength of $322 \mathrm{~nm}$. The dissolution profiles were compared using the values of dissolved benznidazole at 30,60 and $120 \mathrm{~min}^{16}$

The dissolution data were fitted to several kinetics models such as zero-order and first-order equations, Higuchi, Korsmeyer-Peppas, Hixson-Crowel, and Weibull models using the Microsoft Excel ${ }^{\oplus} .{ }^{17}$ The interpretation of the most suitable mathematical model for each formulation was performed by analyzing the adjusted coefficient $\left(\mathrm{R}^{2}\right)$ and the coefficient of correlation (R).

In vitro test of trypanocidal compounds adopting $\beta$-galactosidase-transfected Tulahuen Trypanosoma cruzi strain

The in vitro test of trypanocidal activity was performed as previously described by Romanha et al. ${ }^{18}$ using T. cruzi (Tulahuen strain) expressing the Escherichia coli $\beta$-galactosidase gene. Infective trypomastigote forms were obtained through culture in monolayers of mouse L929 fibroblasts in Roswell Park Memorial Institute (RPMI) 1640 medium ( $\mathrm{pH}$ 7.2-7.4), without phenol red (Gibco BRL, Waltham, MA, USA), containing $10 \%$ fetal bovine serum and $2 \mathrm{mM}$ glutamine. For the bioassay, 4,000 L929 cells in $80 \mu \mathrm{L}$ of supplemented medium were added to each well of a 96-well microtiter plate. After an overnight incubation, 40,000 trypomastigotes in $20 \mu \mathrm{L}$ were added to the cells and the plate was incubated for $2 \mathrm{~h}$. Medium containing parasites that did not penetrate the cells was replaced with $200 \mu \mathrm{L}$ of fresh medium and the plate was incubated for an additional $48 \mathrm{~h}$ to establish infection. The medium was then replaced with solutions of compounds at different concentrations in fresh medium $(200 \mu \mathrm{L})$ and the plate was incubated for $96 \mathrm{~h}$ at $37^{\circ} \mathrm{C}$. After this period, $50 \mu \mathrm{L}$ of $500 \mu \mathrm{M}$ chlorophenol red beta- $D$-galactopyranoside (CPRG) in $0.5 \%$ Nonidet P40 were added to each well and the plate was incubated for $18 \mathrm{~h}$ at $37{ }^{\circ} \mathrm{C}$, after which the absorbance at $570 \mathrm{~nm}$ was measured. Controls with uninfected cells, untreated infected cells, infected cells treated with benznidazole (positive control) or DMSO 1\% were used. The results were expressed as the percentage of $T$. cruzi growth inhibition in compound-tested cells compared to the infected cells and untreated cells. Quadruplicates were run in the same plate and the experiments were repeated at least once.

Determination of cytotoxicity on L929 cells $\left(\mathrm{CC}_{50}\right)$ and selectivity index

For this bioassay, 4,000 L929 cells in $200 \mu \mathrm{L}$ of RPMI-1640 medium (pH 7.2-7.4) (Gibco, Thermo Sci, Waltham, MA, USA) plus $10 \%$ fetal bovine serum and $2 \mathrm{mM}$ 
glutamine were added to each well of a 96-well microtiter plate that was incubated for three days at $37^{\circ} \mathrm{C}$. The medium was then replaced and the cells were exposed to compounds at increasing concentrations starting at 50\% inhibitory concentration $\left(\mathrm{IC}_{50}\right)$ value for T. cruzi. After $96 \mathrm{~h}$ of incubation with the compounds, the alamarBlue ${ }^{\mathrm{TM}}$ was added and the absorbance at 570 and $600 \mathrm{~nm}$ was measured after 4-6 h. Controls with untreated cells and DMSO 1\%-treated cells were evaluated in parallel. Quadruplicates were performed in the same plate and the experiments were repeated at least once. The results were expressed through the difference in the percentage of reduction between treated (TC) and untreated cells (UT), using the following equation:

Difference in reduction $(\%)=\frac{(117.216)\left(\mathrm{Abs}_{570} \mathrm{TC}\right)-(80.586)\left(\mathrm{Abs}_{600} \mathrm{TC}\right)}{(117.216)\left(\mathrm{Abs}_{570} \mathrm{UT}\right)-(80.586)\left(\mathrm{Abs}_{600} \mathrm{UT}\right)} \times 100(2)$

The $\mathrm{IC}_{50}$ on $T$. cruzi and $\mathrm{CC}_{50}$ on $\mathrm{L} 929$ cells were determined by linear curve interpolation and the selectivity index (SI) was calculated by the ratio of $\mathrm{CC}_{50} \mathrm{~L} 929 / \mathrm{IC}_{50}$ T. cruzi.

\section{Statistical analysis}

The results are presented as mean and standard error and were analyzed by an analysis of variance (ANOVA) followed by comparison with the Bonferroni test using GraphPad Prism 5.0 software. ${ }^{19}$ The significance level was $p<0.05$.

\section{Results and Discussion}

\section{Synthesis of PEG 4000 derivatives ${ }^{10-14}$}

The chemistry modification and incorporations of
PEG 4000 (P1), acetylated PEG 4000 (P2) and esterified PEG 4000 (P3) with benznidazole were efficient. The PEG 4000 and its derivatives were soluble in chloroform, dichloromethane and heated acetone. The PEGs incorporated with benznidazole (BP1, BP2 and BP3) were soluble in heated acetone. After the modifications at the PEG chain, it was possible to observe the formation of a homogeneous film in the acetylated PEG 4000 (P2) and a heterogeneous film in the esterified PEG 4000 (P3).

\section{Nuclear magnetic resonance (NMR) analysis}

Nuclear magnetic resonance (NMR) is an effective way for monitoring the modification by polymerization due to its high dispersion of signals. In addition, the interpretation of the spectra allows identifying the monomers forming the polymer and determining how they are distributed. ${ }^{20}$ Thus, from the analysis of the results obtained by this technique, it is possible to establish correlations between structure and material properties. ${ }^{21}$ However, the sensitivity of the instrument to detect the terminal group is the main limitation for this analysis since it is directly correlated to the mass of the polymer that will be measured. The spectrum analysis of the precursor P1 (Figure 1) shows the signals related to the polymer triad. The signals at 60.67 and $72.81 \mathrm{ppm}$ were assigned to carbon and ether carbons, respectively, of the polymer terminal chain. The PEG 4000 monomeric repetition showed an intense and characteristic signal at $70.25 \mathrm{ppm}$. The monitoring of functionalization reactions was done by modifying the chemical environment that led to the differentiation of the signal displacement in the terminal chain and the appearance of new signals in the spectrum.

Figure 2 shows the ${ }^{13} \mathrm{C}$ NMR spectrum of the

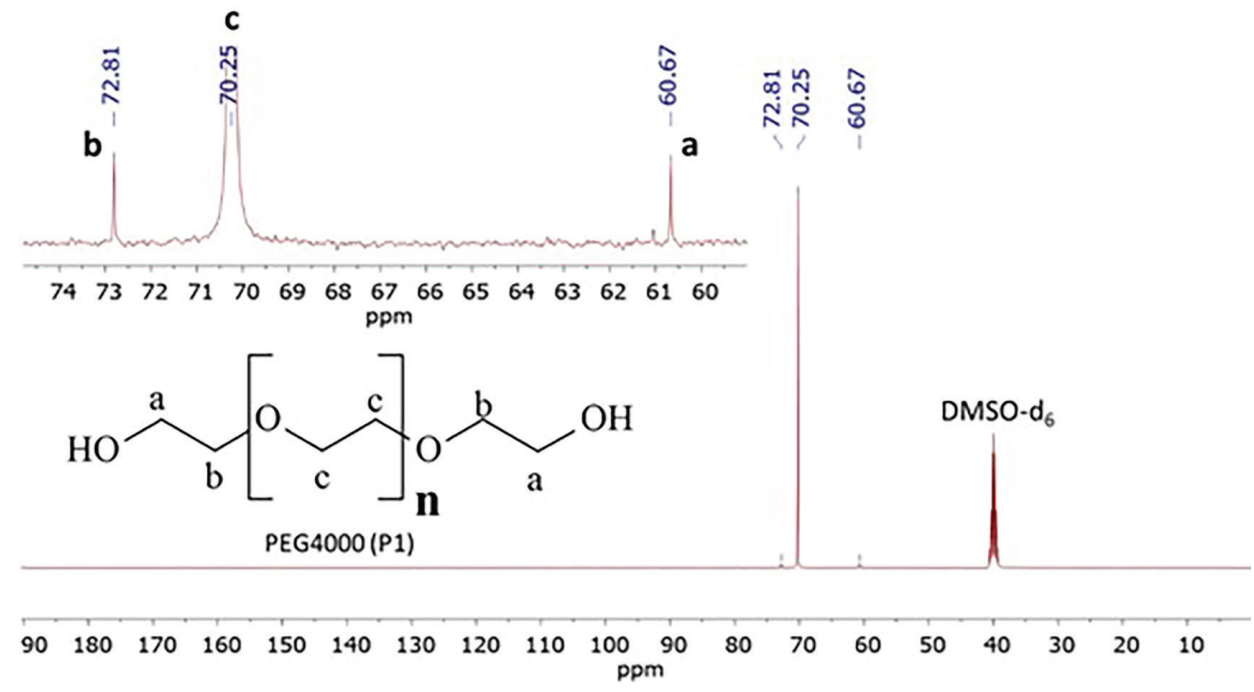

Figure 1. ${ }^{13} \mathrm{C}$ NMR spectrum $\left(100 \mathrm{MHz}, \mathrm{DMSO}-d_{6}\right)$ of PEG $4000(\mathrm{P} 1)$. 
PEG 4000 (P1) acetylation product, acetylated PEG 4000 (P2). The characteristic signals of the reaction product are present in the spectrum. The appearance of a signal at $21.14 \mathrm{ppm}$ attributed to the methyl group $\alpha$ to the carboxyl of the ester formed (170.79 ppm) was observed. The acetylation led to a modification in the terminal chain of the precursor leading to a differentiation of the carbons when compared to PEG 4000. There were two more signs in the region of the carbinolic carbons attributed to the $\mathrm{CH}_{2}$ of the ester $(63.66 \mathrm{ppm})$ and $\mathrm{CH}_{2}$ of the ether $(68.72 \mathrm{ppm})$. As can be seen, the signals referring to the terminal carbons of the precursor (60.67 and $72.81 \mathrm{ppm}$ ) are still present, indicating that there was no complete functionalization. The esterified PEG 4000 (P3) polymer synthesized from PEG 4000 showed a similar structure to $\mathrm{P} 2$.

Benznidazole was also characterized by ${ }^{13} \mathrm{C}$ NMR. All signals related to the molecule have been assigned. In the Figure 3, three distinct regions can be observed. The first one refers to methylene carbons $\left(\mathrm{N}-\mathrm{CH}_{2}-\right)$ at 42.77 and $52.06 \mathrm{ppm}$. The second one is characterized by the aromatic $\mathrm{sp}^{2}$ carbon region of 127.41-145.42 ppm, featuring the phenyl group and nitro-imidazole. The region with the most unblocked signal in the spectrum was attributed to the amide carboxyl at $166.13 \mathrm{ppm}$. When the spectra of the free benznidazole is compared to encapsulated formulations based on synthesized polymers (Figure 4), all signals referring to the reference drug were observed, indicating its incorporation into the particles.
Atomic force microscopy (AFM)

In all cases, considerable changes on the film organization can be seen, produced by a modification of the polymer chain and/or by incorporation process as shown in Figure 5.

The observed films in Figure 5 are probably the result of the collapse of PEG microparticles upon contact with the mica substrate used for AFM imaging (in a similar way to the collapse of soap bubbles when in contact with a surface). Such process, therefore, precludes the observation of intact and whole PEG microcapsules via AFM.

\section{Determination of zeta potential}

In order to predict the stability of the formulations, zeta potential was determined. This parameter is related to repulsion forces between the particles and high values (in module) can reduce the appearance of possible instability processes..$^{22}$ The acetylation of PEG 4000 (BP2) favored the increase in the zeta potential of the microparticles, but no statistical difference was observed between raw (BP1) and esterified polymer (BP3) (Table 1). Although a value greater than $|30| \mathrm{mV}$ is recommended for stable formulations, steric stabilization provided by the bulky surface groups of PEG would be enough to ensure stability of the product. These low results can also be associated with the properties of PEG 4000 that has no surface charge. ${ }^{23}$ In addition, polymeric particles can be stabilized by electrostatic and/or steric stabilization using surfactants, such as poly(vinyl alcohol). ${ }^{24}$

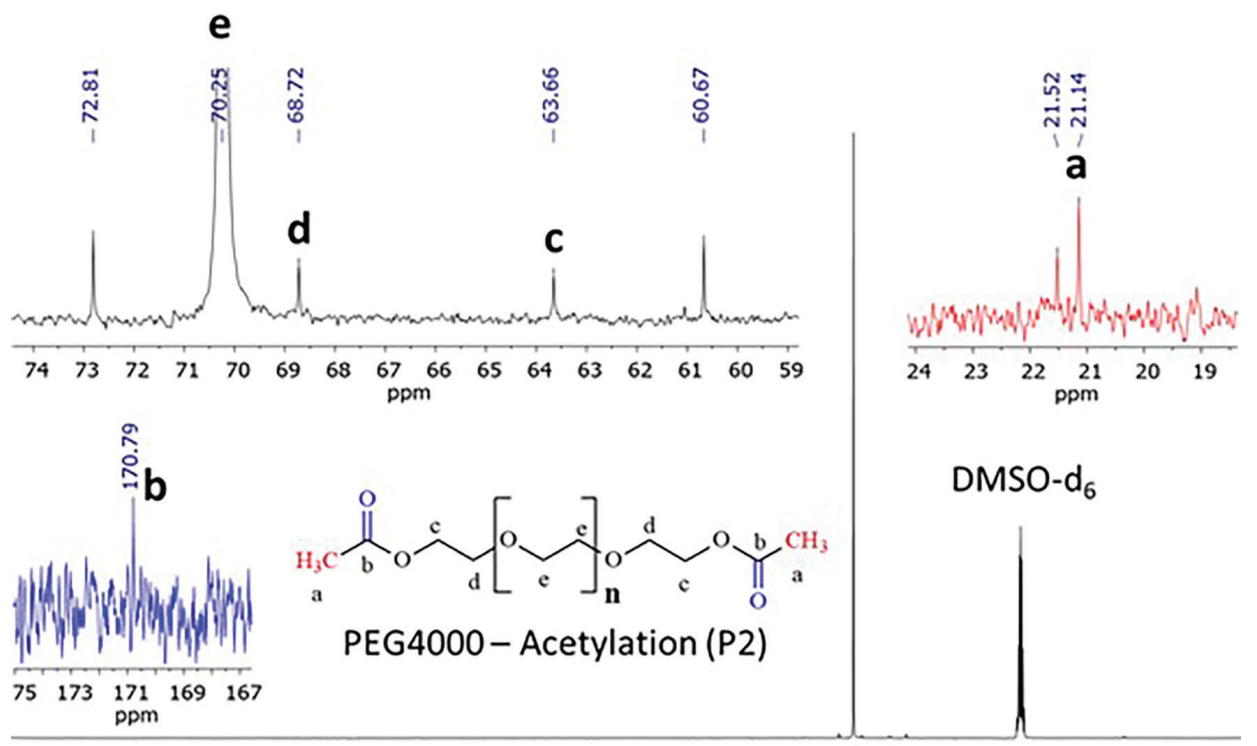

$\begin{array}{llllllllllllllllllllllllllllllllllll}220 & 210 & 200 & 190 & 180 & 170 & 160 & 150 & 140 & 130 & 120 & 110 & 100 & 90 & 80 & 70 & 60 & 50 & 40 & 30 & 20 & 10\end{array}$

Figure 2. ${ }^{13} \mathrm{C}$ NMR spectrum (100 MHz, DMSO- $d_{6}$ ) of acetylated PEG 4000 (P2). 


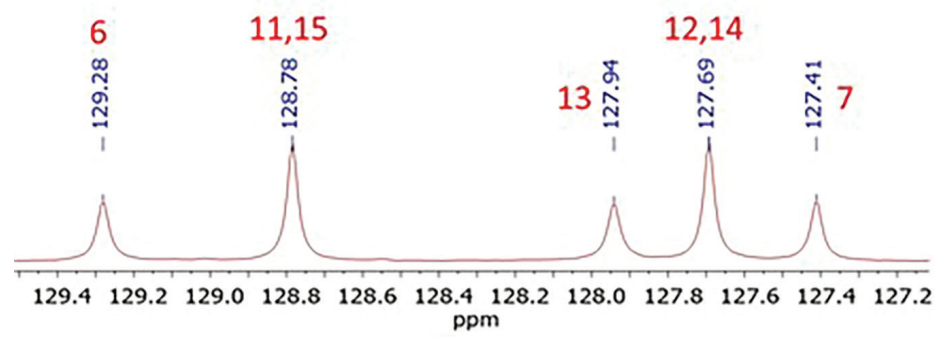<smiles>O=C(Cn1ccnc1[N+](=O)[O-])NCc1ccccc1</smiles>

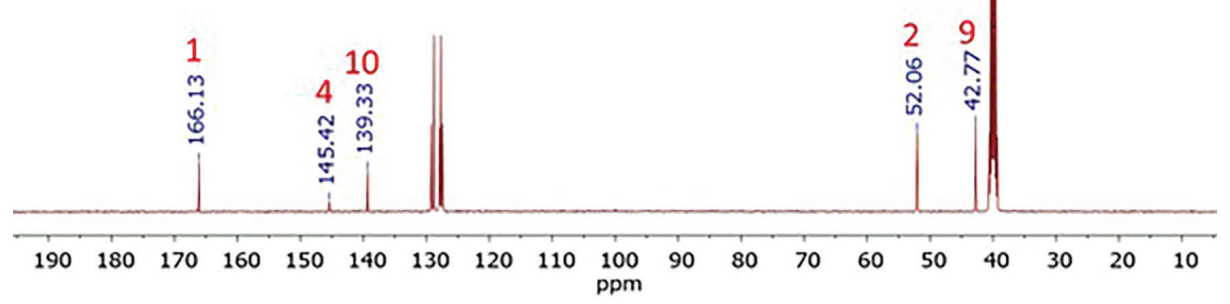

Figure 3. ${ }^{13} \mathrm{C}$ NMR spectrum (100 MHz, DMSO- $d_{6}$ ) of benznidazole (BZ).

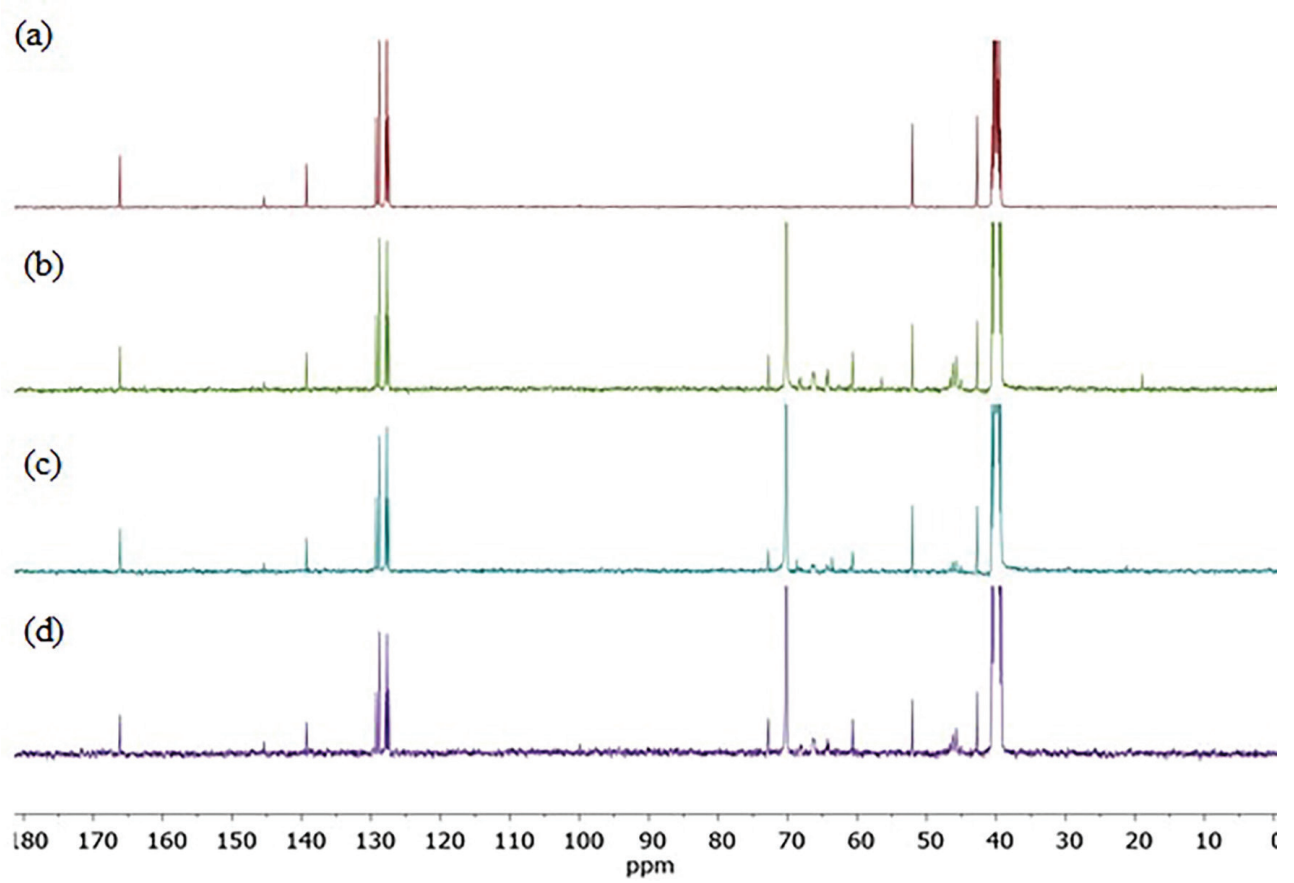

Figure 4. ${ }^{13} \mathrm{C}$ NMR spectrum (100 MHz, DMSO- $d_{6}$ ) of (a) benznidazole, (b) PEG 4000 loaded with benznidazole (BP1), (c) acetylated PEG 4000 loaded with benznidazole (BP2) and (d) esterified PEG 4000 loaded with benznidazole (BP3).

\section{Determination of encapsulation efficiency}

Before determination of encapsulation efficiency (EE), absorbance scanning analysis confirmed that the solvent and particle components do not interfere in the range of maximum wavelength for benznidazole (Figure 6a). Then, entrapment efficiency of benznidazole was determined and values of $39.48,30.14$ and $32.85 \%$ were found for BP1, BP2 and BP3, respectively (Table 1). The loss of hydrophobic drugs from the polymeric walls may occur during the solvent evaporation process and $\mathrm{EE}$ values around $60 \%$ are generally demonstrated for this condition. ${ }^{25-27}$

According to Barrera et al. ${ }^{28}$ chitosan microparticles loaded with benznidazole showed EE between 45 and $78 \%$ 

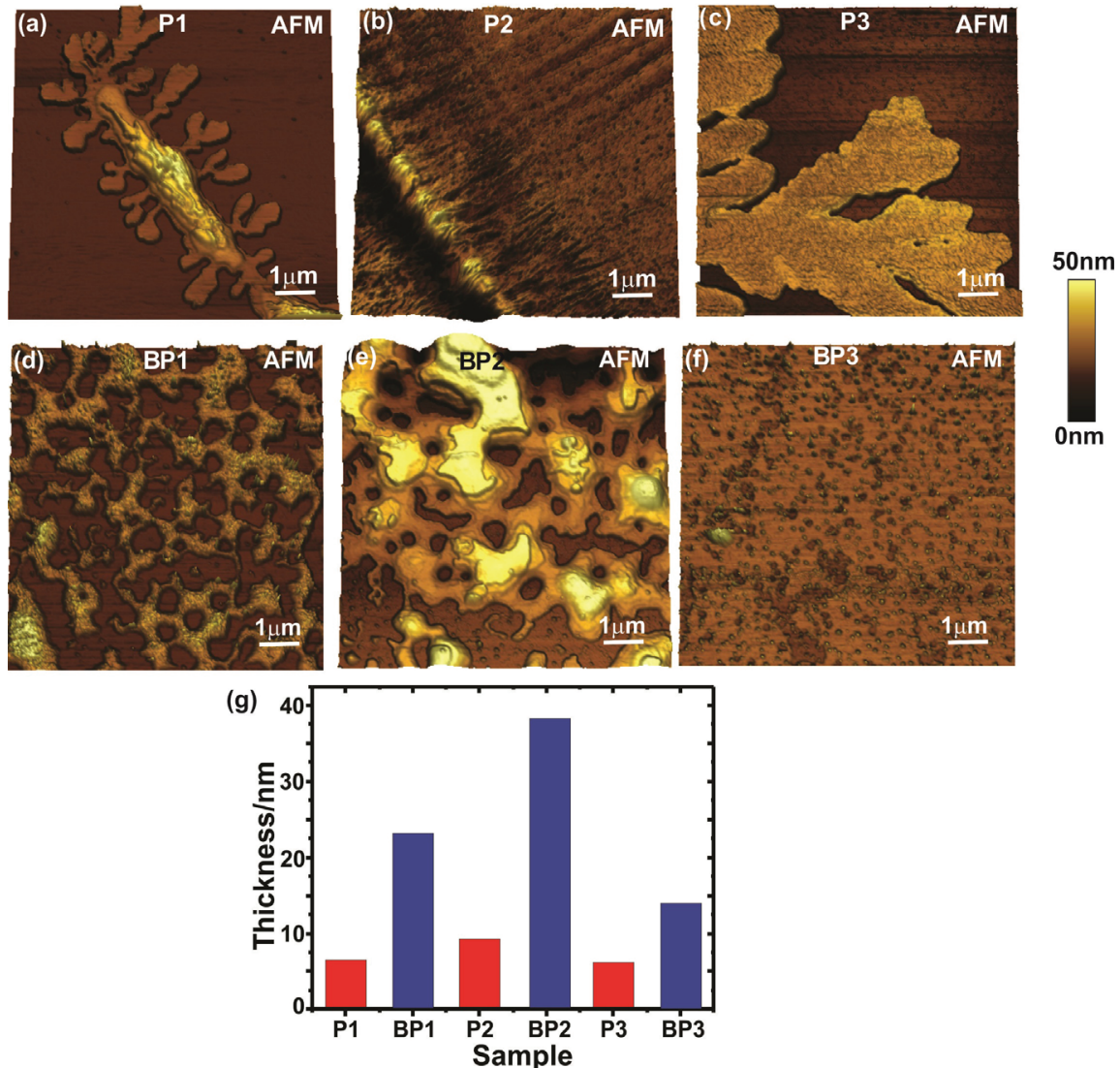

Figure 5. Atomic force microscopy (AFM) image of (a) PEG (P1), (b) acetylated PEG 4000 (P2), (c) esterified PEG 4000 (P3), (d) PEG 4000 loaded with benznidazole (BP1), (e) acetylated PEG 4000 loaded with benznidazole (BP2), (f) esterified PEG 4000 loaded with benznidazole (BP3) and (g) the thickness of films before (red bars) and after incorporation process (blue bars).

Table 1. Zeta potential and encapsulation efficiency (EE) of the benznidazole-loaded microparticles obtained from raw and modified PEG 4000

\begin{tabular}{lcc}
\hline Polymer & Zeta potential $/ \mathrm{mV}$ & EE $/ \%$ \\
\hline BP1 & $-14.83 \pm 3.66$ & $39.48 \pm 0.49$ \\
BP2 & $-25.54 \pm 5.11^{\mathrm{a}}$ & $30.14 \pm 7.85^{\mathrm{a}}$ \\
BP3 & $-14.13 \pm 3.77$ & $32.85 \pm 2.69^{\mathrm{a}}$ \\
\hline
\end{tabular}

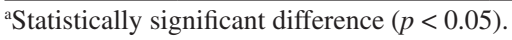

depending on the method. In another study, ${ }^{29}$ values above $78 \%$ were found for benznidazole EE of microstructures obtained from Eudragit ${ }^{\circledast}$. Although our results showed a moderate percentage of benznidazole incorporated into the particles, it is expected that lower doses of the drug will be administered. This fact is related to the improvement in the bioavailability of the drug, which can be achieved by increasing the dissolution rate of benznidazole when encapsulated.

\section{Determination of dissolution profile}

It is already known that the low water solubility of benznidazole $\left(0.4 \mathrm{mg} \mathrm{mL}^{-1}\right)$ impairs its oral bioavailability and the development of formulations can overcome this problem and consequently reduce the administered dose. Dissolution studies were performed at $\mathrm{pH} 3$ and 7.3 to simulate the physiological properties for oral and intravenous administration, respectively. According to Figure 6, both modified polymers increased the benznidazole dissolution rate when compared to the raw drug in acidic conditions. Although similar values of dissolved benznidazole were observed for all samples at 30 and $60 \mathrm{~min}$, greater drug solubility was confirmed after $120 \mathrm{~min}$ for the microparticles obtained from the modified PEG 4000, reaching peaks of more than $90 \%$. This result can be related to the medium conditions, since the acidic $\mathrm{pH}$ favors the hydrolysis of the polymers (BP2 and BP3) and consequently a greater drug release. According to Ventura et al. ${ }^{30}$ cyclodextrin formulation based on ester-binding showed greater drug release in media simulating gastro-intestinal fluids than in $\mathrm{pH}$ 7.4. On the other hand, no improvement for this parameter was observed after incorporation of benznidazole in microparticles when a neutral medium was used, since polymers hydrolysis occurs in an acidic or basic medium. ${ }^{30}$

In this way, dissolution efficiency was determined for benznidazole micronized particle stabilized with several 

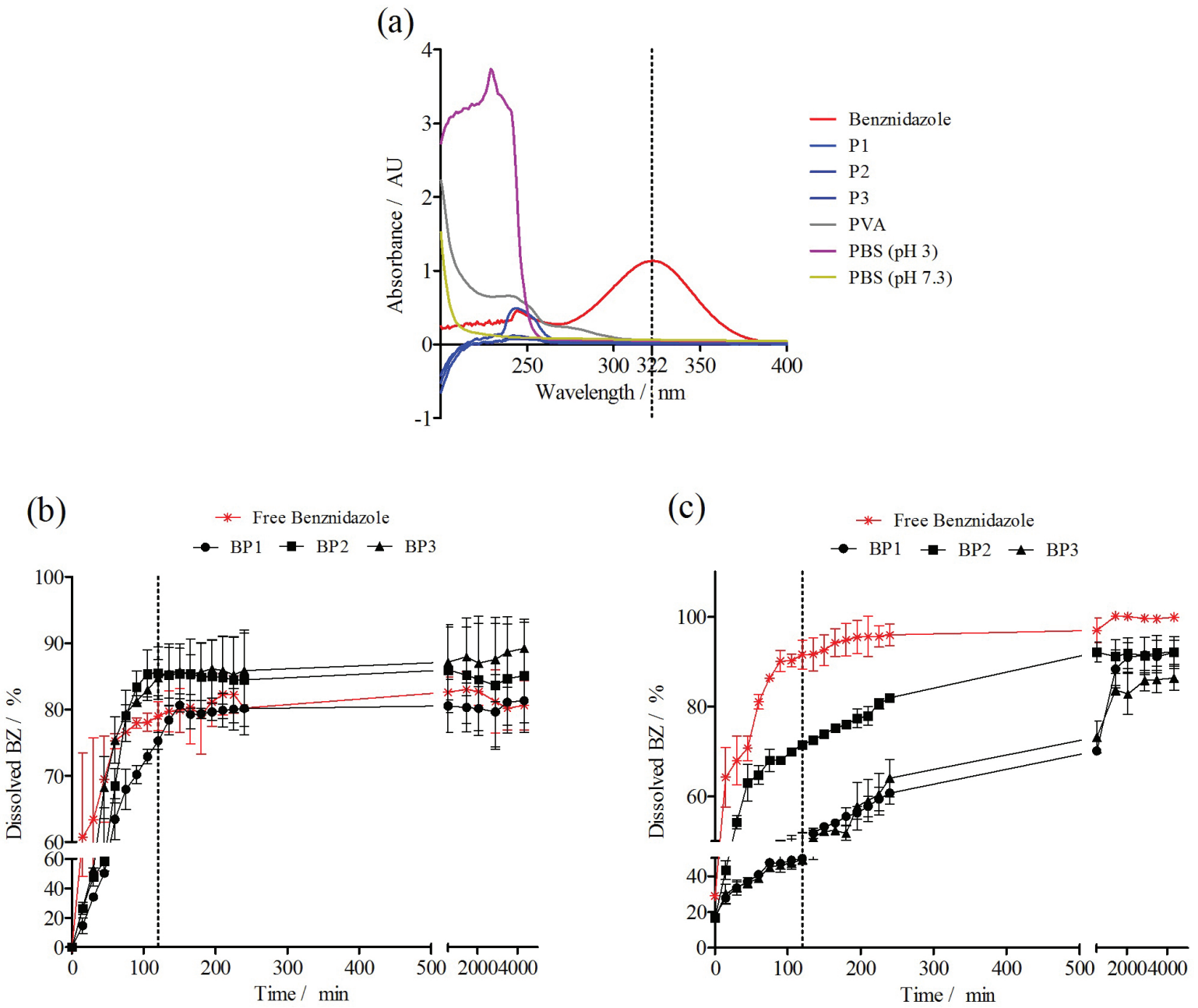

Figure 6. Absorbance spectrum (a) by spectrophotometric method for different samples: benznidazole, polymers (P1-P3), polyvinyl alcohol (PVA) and phosphate buffered saline (PBS). Dissolution profile of free benznidazole and benznidazole-loaded microparticles obtained from different polymers (BP1BP3) using phosphate buffer solution at $\mathrm{pH} 3$ (b) and $\mathrm{pH} 7.3$ (c).

polymers (F50, K100, E10m, PEG and hydroxyethylcellulose (HEC)) and values above 60 and $20 \%$ were found for PEG 4000 and untreated drug, respectively. ${ }^{31}$ Similarly, dissolution rates from 70 to $99 \%$ were observed for chitosan microparticles obtained from two methods in three different mediums, enhancing the solubility of raw benznidazole. ${ }^{32}$ This result is also consistent with the work of Seremeta et al. ${ }^{31}$ who demonstrated that microparticles based on Eudragit ${ }^{\circledast}$ were able to improve the dissolution profile of this drug from the beginning of the test. ${ }^{29}$ However, the maximum percentage of benznidazole dissolved at $120 \mathrm{~min}$ was $52 \%$, which is below the average of $85 \%$ found for pegylated formulations. This result suggests the efficiency of acetylation and esterification of the polymer as an alternative to improve the dissolution characteristic of hydrophobic drugs orally administered and it can be related to the reduction of their crystalline structure.

Data of the dissolution profiles were fitted to different equations and kinetic models to explain the release kinetics of benznidazole-loaded microparticles obtained from different polymers. The kinetic models used were a zero-order and first-order equations, Higuchi, KorsmeyerPeppas, Hixson-Crowel, and Weibull models..$^{33}$ The best fit with the highest correlation $\mathrm{R}$ and adjusted $\mathrm{R}^{2}$ coefficients were shown by Higuchi and Korsmeyer-Peppas (Table 2).

For Higuchi model, the drug release can be defined as the mass transfer of drug molecules from the dosage form to the surrounding medium. Higuchi model describes the release of drugs as the square root of time based on the Fickian diffusion and it has a large application in polymeric matrix systems..$^{33}$ Thus drug release mechanism was assumed to be diffusion controlled.

Korsmeyer-Peppas is also often applied for the analysis of the release profile of drugs in polymeric systems. This model is based on the Fick's Law. It is used to describe the release of the solute when the prevailing mechanism is a combination of drug diffusion, Fickian transport, and in case II transport, non-Fickian, controlled by the 
Table 2. Adjusted coefficient $\left(\mathrm{R}^{2}\right)$ and coefficient of correlation $(\mathrm{R})$ kinetic obtained by Higuchi and Korsmeyer-Peppas model in $\mathrm{pH} 3.0$ and $\mathrm{pH} 7.0$

\begin{tabular}{|c|c|c|c|c|c|c|c|c|}
\hline \multirow{2}{*}{ Polymer } & \multicolumn{2}{|c|}{$\mathrm{pH} 3.0^{\mathrm{a}, \mathrm{b}}$} & \multicolumn{2}{|c|}{$\mathrm{pH} 7.0^{\mathrm{a}, \mathrm{b}}$} & \multicolumn{2}{|c|}{$\mathrm{pH} 3.0^{\mathrm{b}, \mathrm{c}}$} & \multicolumn{2}{|c|}{$\mathrm{pH} 7.0^{\mathrm{b}, \mathrm{c}}$} \\
\hline & $\mathrm{R}$ & $\mathrm{R}^{2}$ & $\mathrm{R}$ & $\mathrm{R}^{2}$ & $\mathrm{R}$ & $\mathrm{R}^{2}$ & $\mathrm{R}$ & $\mathrm{R}^{2}$ \\
\hline $\mathrm{BP} 1$ & $0.9998^{\mathrm{a}}$ & $0.9997^{\mathrm{a}}$ & $0.9991^{\mathrm{a}}$ & $0.9982^{a}$ & $0.9965^{\mathrm{c}}$ & $0.9930^{\mathrm{c}}$ & $0.9988^{c}$ & $0.9977^{\mathrm{c}}$ \\
\hline $\mathrm{BP} 2$ & $0.9947^{\mathrm{a}}$ & $0.9897^{\mathrm{a}}$ & $0.9728^{\mathrm{a}}$ & $0.9875^{\mathrm{a}}$ & $0.9915^{\mathrm{c}}$ & $0.9830^{\mathrm{c}}$ & $0.9906^{c}$ & $0.9813^{\mathrm{c}}$ \\
\hline BP3 & $0.9869^{\mathrm{a}}$ & $0.9740^{\mathrm{a}}$ & $0.9824^{\mathrm{a}}$ & $0.9963^{\mathrm{a}}$ & $0.9837^{c}$ & $0.9977^{\mathrm{c}}$ & $0.9977^{\mathrm{c}}$ & $0.9955^{\mathrm{c}}$ \\
\hline
\end{tabular}

${ }^{a}$ Coefficient $\left(\mathrm{R}^{2}\right)$ and coefficient of correlation (R) by Higuchi model; bobtained from the publication Costa et al. ${ }^{33}{ }^{\mathrm{c}}$ coefficient $\left(\mathrm{R}^{2}\right)$ and coefficient of correlation (R) Korsmeyer-Peppas model.

relaxation of polymer chains. ${ }^{33}$ Due to the fact that drug release in this study occurs in a one-dimensional way and that the system length/thickness relation is by far more than 10 , following the Korsmeyer-Peppas ${ }^{33}$ indications, this equation represents a good choice. The calculated values of $\mathrm{n}$ of the different samples were approximately 0.900 . According to this model, the value of $\mathrm{n}$ identifies the release mechanism of drug. In this study it indicated anomalous transport. ${ }^{33}$ Therefore, in this study the diffusional release was assumed to follow anomalous transport.

\section{Evaluation of in vitro anti-Trypanosoma cruzi activity, in vitro cytotoxicity and selectivity index}

The polymers unloaded and loaded with benznidazole were evaluated for their trypanocidal activity on different T. cruzi evolutionary forms and for cytotoxicity in L929 murine fibroblasts as well. ${ }^{28,34}$ This approach is in accordance with the Drugs for Neglected Diseases Initiative guidelines (DNDi) and has the advantage of evaluating trypanocidal activity in the most relevant forms of $T$. cruzi, and, at the same time, evaluating the cytotoxicity of different compounds in cells. ${ }^{18,35}$ Free benznidazole (positive control) provided an $\mathrm{IC}_{50}(50 \%$ inhibitory concentration) of $0.4 \mu \mathrm{g} \mathrm{mL} \mathrm{m}^{-1}$ and showed a SI of $>1250$. This result was used as a benchmark value for assessing the potency and selectivity of the polymers with benznidazole. The polymers (P1, P2 and P3) without benznidazole (formulation negative control) were inactive against $T$. cruzi. According to Romanha et al. ${ }^{18}$ microparticles BP1, BP2 and BP3 (Table 3) were active and highly selective (SI $\geq 50$ ). The current formulations have 5.2 times less benznidazole than the positive control benznidazole (BZ). Considering this fact, we can say that polymers were able to maintain the trypanocidal activity of benznidazole.

Reducing the dose of benznidazole and maintaining its trypanocidal activity is very important, since the increase in the intensity of adverse reactions to benznidazole is linked to the dose, being more frequent and intense with higher doses. ${ }^{36}$ The adverse effects of benznidazole (hypersensitivity, digestive intolerance, anorexia, asthenia, headache, sleep disorders, neuropathy and depression of the immune system as a result of bone marrow depression) lead to treatment dropout in about 9.0 to $29.0 \%$ of cases, which reinforces the importance of reducing its dose in new treatments. ${ }^{37-40}$

\section{Conclusions}

The efficiency loss of benznidazole is largely associated with its low oral bioavailability. The development of formulations that circumvent these disadvantages would be of great value to reduce the doses of the drug and consequently its adverse effects. Microparticles developed from modified PEG 4000 were able to increase the solubility of benznidazole and consequently reproduce and even increase the trypanocidal effect of this drug from lower concentrations when compared to its conventional form. In addition, particles obtained from modified

Table 3. Trypanocidal activity, cytotoxicity and selectivity index

\begin{tabular}{lccc}
\hline Polymer & $\mathrm{IC}_{50}{ }^{\mathrm{a}} /\left(\mu \mathrm{g} \mathrm{mL}{ }^{-1}\right)$ & $\mathrm{CC}_{50}{ }^{\mathrm{b}} /\left(\mu \mathrm{g} \mathrm{mL}^{-1}\right)$ & $\mathrm{SI}^{\mathrm{c}}$ \\
\hline BP1 & $2.2(0.42)^{\mathrm{d}} \pm 0.8$ & $>1,200.0(230.8)^{\mathrm{d}}$ & $>545.4^{\mathrm{c}}$ \\
BP2 & $3.5(0.67)^{\mathrm{d}} \pm 2.6$ & $>1,200.0(230.8)^{\mathrm{d}}$ & $>342.8^{\mathrm{c}}$ \\
BP3 & $1.6(0.31)^{\mathrm{d}} \pm 1.1$ & $>1,200.0(230.8)^{\mathrm{d}}$ & $>750.0^{\mathrm{c}}$ \\
Benznidazole & $0.4 \pm 0.1$ & $>500.0$ & $>1,250.0$ \\
\hline
\end{tabular}

${ }^{\mathrm{a}}$ Trypanocidal activity $\mathrm{IC}_{50}\left(50 \%\right.$ inhibitory concentration); ${ }^{\mathrm{b}} \mathrm{CC}_{50}$ : $50 \%$ cytotoxic concentration determined using mammalian L929 cells; ${ }^{\mathrm{c}} \mathrm{SI}$ : selectivity index calculated from $\mathrm{CC}_{50} / \mathrm{IC}_{50}$; ${ }^{\text {d }}$ benznidazole concentration in the formulation. 
PEG 4000 also showed a higher zeta potential than the crude polymer, which may suggest greater stability for these formulations. Thus, the efficiency of polymer modification was demonstrated by NMR analysis and emerges as a new safe alternative for the treatment of CD.

\section{Acknowledgments}

The authors gratefully acknowledge the generous financial support from the Federal University of Ouro Preto (UFOP), FAPEMIG, and the Conselho Nacional de Desenvolvimento Científico e Tecnológico (CNPq). The authors are thankful to the Technological Development Program of Tools for Health-PDTIS-FIOCRUZ for the use of its facilities.

\section{Author Contributions}

Lucas R. D. Sousa, Maria Luíza S. Azevedo and Dayana F. Rocha were responsible for data curation, investigation, resources and visualization; Ângela L. Andrade for conceptualization, investigation and validation; Orlando D. H. dos Santos and Janaína B. Seibertfor formal analysis funding acquisition, validation, visualization and writing original draft; Luciano R. Pereira for conceptualization, data curation, and visualization; Tatiane R. Amparo, Paula M. A. Vieira and Cláudia M. Carneiro for data curation and resources; Ana Paula M. Barboza and Bernardo R. A. Neves for data curation, formal analysis funding acquisition, investigation, resources and validation and writing original draft;Policarpo A. Sales Jr. and Silvane M. F. Murta for data curation, resources and writing-review; Kátia M. Novack for visualization and writing original draft; Viviane M. R. dos Santos for investigation, project administration resources, writing original draft and writing-review and editing.

\section{References}

1. Diogo, G. M.; Andrade, J. S.; Sales Jr., P. A.; Murta, S. M. F.; dos Santos, V. M. R.; Taylor, J. G.; Molecules 2020, 25, 14.

2. Kropf, S. P.; Massarani, L.; Carlos Chagas: A Ciência para Combater Doenças Tropicais; Casa de Oswaldo Cruz/Fiocruz: Rio de Janeiro, 2009.

3. http://portalarquivos.saude.gov.br/images/pdf/2015/ agosto/03/2014-020..pdf, accessed in February 2021.

4. Pinheiro, E.; Brum-Soares, L.; Reis, R.; Cubides, J.; Rev. Soc. Bras. Med. Trop. 2017, 50, 296.

5. Karolewicz, B.; Saudi Pharm. J. 2016, 24, 525.

6. Arrúa, E. C.; Seremeta, K. P.; Bedogni, G. R.; Okulik, N. B.; Salomon, C. J.; Acta Trop. 2019, 198, 105080.
7. Alencar, G. O.; Bezerra, C. A.; Scherf, J. R.; da Silva, J. F.; Barreto, M. F. R.; Alves, M. P.; Mendes, R. C.; Rev. e-ciência 2018, 6,5 .

8. Oliveira, R. B.; Rev. Eletronica Farm. 2006, 3, 29.

9. Farmacopeia Brasileira, vol. 2; ANVISA: Brasília, 2010.

10. Santos, V. M. R.; Novack, K. M.; Silveira, B. M.; Rosa, J. S.; Macromol. Symp. 2014, 343, 78.

11. Santos, V. M. R.; Novack, K. M.; de Sousa, D. V. M.; Oliveira, S. R.; Donnici, C. L.; Polimeros 2015, 25, 19.

12. Silveira, B. M.; Novack, K. M.; Marcondes, H. C.; Santos, V. M. R.; Macromol. Symp. 2018, 378, 1700066.

13. Azevedo, M. L. S.; Silveira, B. M.; Moneiro, K. N.; dos Santos, V. M. R.; Macromol. Symp. 2018, 381, 1800145.

14. Nascimento, L. G.; Lopes, S. A.; Teodolino, A. B. L.; Novack, K. M.; Barboza, A. P. M.; Nevas, B. R. A.; Azevedo, M. L. S.; Sousa, L. R. D.; dos Santos, V. M. R.; Quim. Nova 2020, 43, 685.

15. Pérez-Molina, J. A.; Molina, I.; Lancet 2018, 391, 82.

16. Kwon, H. Y.; Lee, J. Y.; Choi, S. W.; Jang, Y.; Kim, J. H.; Colloids Surf., A 2001, 182, 123.

17. Gates III, W. H.; Allen, P. G.; Microsoft Excel software; Microsoft Corporation, Redmond, Washington, United States, 2013.

18. Romanha, A. J.; de Castro, S. L.; Soeiro, M. N. C.; LannesVieira, J.; Ribeiro, I.; Talvani, A.; Bourdin, B.; Blum, B.; Olivieri, B.; Zani, C.; Spadafora, C.; Chiari, E.; Chatelain, E.; Chaves, G.; Calzada, J. E.; Bustamante, J. M.; Freitas-Junior, L.-H.; Romero, L. I.; Bahia, M. T.; Lotrowska, M.; Soares, M.; Andrade, S. G.; Armstrong, T.; Degrave, W.; Andrade, Z. A.; Mem. Inst. Oswaldo Cruz 2010, 105, 233.

19. Radushev, D.; Prism 5 for Windows, version 5.01; GraphPad Software, United States, 2007.

20. Pillai, O.; Panchagnula, R.; Curr. Opin. Chem. Biol. 2001, 5, 447.

21. Oréfice, R. L.; Pereira, M. M.; Mansur, H. S.; Biomateriais: Fundamentos e Aplicações; Cultura Médica: Rio de Janeiro, 2006, ch. 6 .

22. https://www.sigmaaldrich.com/technical-documents/articles/ material-matters/polymer-analysis-by.html, accessed in February 2021.

23. Diehl, B. In NMR Spectroscopy in Pharmaceutical Analysis; Holzgrabe, U.; Wawer, I.; Diehl, B., eds.; Elsevier Science: Poland, 2008, ch. 2.

24. Acevedo, F. A.; Salvia, T. L.; Rojas, G. M. A.; Martín, B. O.; Food Hydrocolloids 2015, 47, 168.

25. Capek, I.; Adv. Colloid Interface Sci. 2002, 99, 77.

26. Crucho, C. I. C.; Barros, M. T.; Polymer 2015, 68, 41.

27. Mobarak, D. H.; Salah, S.; Elkheshen, S. A.; Pharm. Dev. Technol. 2014, 19, 891.

28. Barrera, M. G.; Tejada G.; Leonardi, D.; Lamas, M. C.; Salomón, C. J.; AAPS PharmSciTceh 2020, 21, 112. 
29. Budhian, A.; Siegel, S. J.; Winey, K. I.; Int. J. Pharm. 2007, 336, 367.

30. Ventura, C. A.; Paolino, D.; Pedotti, S.; Pistarà, V.; Corsaro, A.; Puglisi, G.; J. Drug Targeting 2003, 11, 233.

31. Seremeta, K. P.; Arrúa, E. C.; Okulik, N. B.; Salomon, C. J.; Colloids Surf., B 2019, 177, 169.

32. Maximiano, F. P.; de Paula, L. M.; Figueiredo, V. P.; de Andrade, I. M.; Talvani, A.; Sá-Barreto, L. C.; Bahia, M. T.; Cunha-Filho, M. S. S.; Eur. J. Pharm. Biopharm. 2011, 78, 377.

33. Costa, P.; Lobo, J. M. S.; Eur. J. Pharm. Sci. 2001, 13, 123.

34. Elias, P. R.; Coelho, G. S.; Xavier, V. F.; Sales Jr., P. A.; Romanha, A. J.; Murta, S. M. F.; Carneiro, C. M.; Taylor, J. G.; Molecules 2016, 21, 1342.

35. de Souza, A. A. N.; Xavier, V. F.; Coelho, G. S.; Sales Jr., P. A.; Romanha, A. J.; Murta, S. M. F.; Carneiro, C. M.; Taylor, J. G.; J. Braz. Chem. Soc. 2018, 29, 269.
36. Dias, J. C. P.; Coura, J. R.; Clínica e Terapêutica da Doença de Chagas: uma Abordagem Prática para o Clínico Geral; FIOCRUZ: Rio de Janeiro, 1997.

37. Coura, J. R.; de Castro, S. L.; Mem. Inst. Oswaldo Cruz 2002, $97,3$.

38. Coura, J. R.; Dias, J. C. P.; Mem. Inst. Oswaldo Cruz 2009, 104, 31.

39. Coura, J. R.; Junqueira, A. C. V.; Mem. Inst. Oswaldo Cruz 2015, 110, 825 .

40. Seibert, J. B.; Viegas, J. S. R.; Almeida, T. C.; Amparo, T. R.; Rodrigues, I. V.; Lanza, J. S.; Frézard, F. J. G.; Soares, R. D. O. A.; Teixeira, L. F. M.; Souza, G. H. B.; Vieira, P. M. A.; Barichello, J. M.; Santos, O. D. H.; J. Nat. Prod. 2019, 82, 3208.

Submitted: October 30, 2020 Published online: February 8, 2021 